\title{
Certificação de Qualidade em Engenharia de Requisitos
}

\author{
Edna Pacheco Zanlorenci ${ }^{1,2}$, Robert Carlisle Burnett ${ }^{1}$ \\ e-mail: [ednapz@pr.gov.br] [robert@ppgia.pucpr.br] \\ ${ }^{1}$ Pontifícia Universidade Católica do Paraná, PUC-PR (www.pucpr.br) \\ PPGIA - Programa de Pós-graduação em Informática Aplicada \\ Rua Imaculada Conceição, 1155, Prado Velho, cep 80215-901, Curitiba, Paraná, BRASIL \\ ${ }^{2}$ Companhia de Informática do Paraná - CELEPAR (www.celepar.gov.br) \\ Rua Mateus Leme, 1561 - Centro Cívico, cep 80510-030 - Curitiba, Paraná, BRASIL
}

\begin{abstract}
Resumo
A excelência para a certificação de qualidade é um dos objetivos das organizações. No mundo globalizado, a competitividade é um fator de sobrevivência do negócio. Na área de software não é diferente, seja no cenário acadêmico, na indústria, na pesquisa \& desenvolvimento ou no mercado. Para a Engenharia de Requisitos, a questão é: Como garantir que os processos e os produtos tenham a garantia de qualidade requerida e adequada às prioridades dos stakeholder? O artigo propõe abordar os processos e técnicas, nas fases do ciclo de vida de projeto, pela aplicação sistemática de um script e um checklist para habilitar a validação dos produtos e promover a certificação de qualidade em Engenharia de Requisitos.
\end{abstract}

Palavras-chave: certificação, ciclo de vida, modelo, processo, qualidade, Engenharia de Requisitos.

\begin{abstract}
The excellency to the quality certification is one of the organizations' goals. In the global world, competitiveness is a survival factor in business. In the software area it is not different, either in the academic, industry, research \& development or market scenery. For the Requirements Engineering (RE) the question is: How to ensure that processes and products have the quality assurance required and adjusted to the stakeholder's priorities? The article proposal is to approach the RE processes and techniques, in the project life-cycle phases, through systematic application of a script and a checklist in the processes, to be able for validate products and to promote the quality certification in $R E$.
\end{abstract}

Keywords: certification, life-cycle, model, process, quality, Requirements Engineering.

\section{Introdução}

A motivação para este trabalho é a demanda por parte da indústria de software em obter a certificação de qualidade na área da Engenharia de Requisitos, de maneira similar aos processos ISO/IEC9000 [9] e CMMI [5].

A idéia é o tratamento de requisitos, aplicado ao contexto de desenvolvimento de projeto de software. Um elemento primordial a considerar é o nível de maturidade da organização na aplicação dos processos da Engenharia de Requisitos [21] (descobrimento, análise, validação, documentação e gerência de requisitos) utilizando-se de técnicas adequadas ao ambiente em estudo e às restrições de negócio.

A abrangência da proposta é visualizar no ciclo de vida de um projeto de software, as fases (demanda, estudo de viabilidade, modelo lógico, modelo físico, construção e 
implantação) onde se aplicam os processos da Engenharia de Requisitos e quais produtos devem ser gerados e avaliados.

A justificativa do esforço é obter sucesso na geração de produtos intermediários com a aplicação dos processos de Engenharia de Requisitos, adequados à situação e ao momento de um contexto de desenvolvimento de projeto de software. E, através dos resultados parciais, possibilitar o processo de avaliação durante todas as fases do projeto, para garantir a qualidade dos processos e dos produtos com a Engenharia de Requisitos.

Existem fatores importantes a considerar no contexto de desenvolvimento de software. Eles referem-se a restrições de prazo, de recursos financeiros, de imposição de tecnologia aplicável a um projeto, entre outros e, conseqüentemente, implicam na adoção de um ciclo de vida adequado para a construção do produto e para a abordagem de tratamento da informação.

Dependendo da complexidade da informação a ser tratada, ou seja, o tamanho do projeto ou a necessidade de conhecimento adicional, as fases que compõem o ciclo de vida de um projeto, podem tornar-se subprojetos de um projeto maior, devendo ser gerenciados como projetos com escopo e objetivos claros e gerar produtos específicos.

O assunto é oportuno porque trata de métodos de desenvolvimento e de processos da Engenharia de Requisitos aplicáveis às exigências de qualidade e assim, obter a descrição mais precisa de requisitos do objeto de contratação para o desenvolvimento de software.

O foco, portanto, é o conhecimento exigido das funcionalidades e das características de qualidade do produto a construir, apoiadas por um roteiro (script) de descrição de requisitos e um guia (checklist) para avaliação de processo e de produto em cada fase de projeto.

$\mathrm{O}$ artigo trata, na parte 2 , o fundamento da abordagem de tratamento dos processos, com o objetivo de garantia de qualidade e certificação em Engenharia de Requisitos. Na parte 3, apresenta por fases do ciclo de vida de um projeto, um modelo para certificação e como aplicá-lo aos processos de requisitos. $\mathrm{Na}$ parte 4 , relata resultados de experimentos com processos da Engenharia de Requisitos e, na parte 5, apresenta conclusão do trabalho.

\section{Fundamento da Abordagem}

Cabe à Engenharia de Requisitos [20], como sub-área da Engenharia de Software, aperfeiçoar os processos para o gerenciamento do ciclo de vida dos requisitos. Deve também propor métodos, ferramentas e técnicas que promovam o desenvolvimento do documento de requisitos, para que este produto retrate o conhecimento do problema, em conformidade à satisfação dos stakeholder e aos padrões de qualidade, relacionado ao que se quer produzir com tecnologia da informação para solução dos problemas ou como oportunidade de negócio.

A certificação de qualidade em Engenharia de Requisitos está ligada à aplicação de seus processos e a avaliação de produtos gerados, em todo o ciclo de vida de um projeto. Isto requer um aperfeiçoamento contínuo dos processos aplicáveis à Engenharia de Software e um grau de maturidade da organização na aplicação dos processos da Engenharia de Requisitos, adequados a situações específicas de projeto. O sucesso do processo de desenvolvimento implica em praticar o uso de padrões de qualidade estabelecidos nas normas ISO/IEC: 9000 [9] (gestão da qualidade) 12207[12] (ciclo de vida de processos) 9126-x [10] / 14598 [11] (qualidade produto), de referência aos modelos de maturidade dos processos CMMI [5], de métodos e técnicas de gestão PDCA (Planning, Do, Control, Action) e PMBOK [16].

$\mathrm{O}$ aperfeiçoamento dos processos de desenvolvimento de software na organização depende do conhecimento teórico e, principalmente, da disposição para mudar. O sucesso da 
mudança será obtido se os esforços da organização estiverem voltados a objetivos comuns de resolver os reais problemas metodológicos.

O uso da Engenharia de Requisitos varia em cada organização. Este assunto é apresentado por Sommerville [18], em seu livro Engineering Requirements, referindo-se a três tipos de guia de referência aplicáveis (básica, intermediária, avançada) e uma lista das dez mais atitudes para o sucesso do processo, indicando a restrição quanto a considerar o nível de maturidade da organização e o tipo de software a desenvolver.

A abordagem proposta indica prioritariamente, o conhecimento do que é para ser feito, do que tratam os requisitos no domínio da aplicação e quais suas formas de apresentação em todo o ciclo de vida de um projeto de software. A idéia está representada na figura.1, sob três aspectos: processos da Engenharia de Requisitos, fases de projeto [22,23] e produtos da fase de projeto.

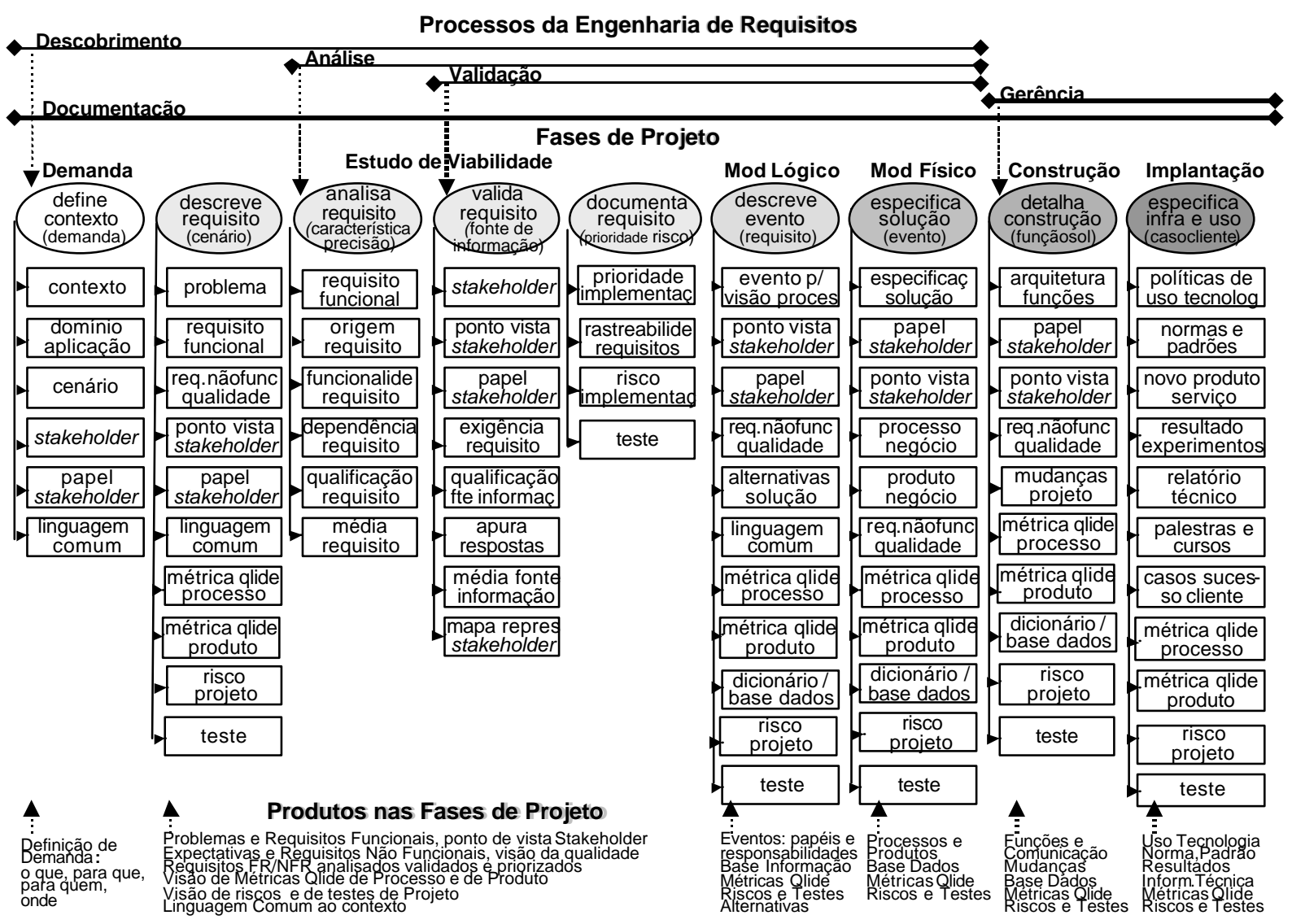

Figura.1 - Processos de RE e Fases de Projeto

A figura.1 apresenta a aplicação dos processos da Engenharia de Requisitos nas fases de projeto. Na parte inicial, apresenta o ciclo dos processos da Engenharia de Requisitos (descobrimento, análise, validação, documentação e gerência de requisitos), relacionados às fases de projeto. Na parte intermediária, apresenta o tratamento do requisito em cada fase de projeto (identificação da demanda, estudo de viabilidade, modelo lógico, modelo físico, construção e implantação), independente do ciclo de vida a ser adotado para o projeto (cascata, incremental, iterativo,...). Na parte final, apresenta os produtos gerados em cada fase de projeto. As figuras elípticas apresentam o objetivo da fase de projeto $\mathrm{e}$ as figuras 
retangulares, a informação obtida e gerada no curso da fase. A representação é uma forma gráfica para sumariar a idéia do modelo.

Os processos da Engenharia de Requisitos e os processos de projeto devem ser tratados num contexto de desenvolvimento de projeto. Independente do ciclo de vida adotado para o desenvolvimento, as fases de projeto estão presentes, o que varia é a intensidade de uso do processo, ou seja, o tempo despendido em cada um e a forma de iteração entre eles.

Do ponto de vista de obtenção da informação, os processos de tratamento de requisitos, têm seus produtos esperados com detalhamento progressivo à medida que evolui o trabalho de desenvolvimento nas fases de projeto. A tabela.1 tem o objetivo de apresentar a ocorrência dos processos de Engenharia de Requisitos, visualizados nas fases de projeto.

Tabela.1 - Relacionamento entre Processos RE e Fases de Projeto

\begin{tabular}{|c|c|c|c|c|c|c|c|c|c|c|c|c|}
\hline $\mathrm{X}$ & \multicolumn{12}{|c|}{ Fases de Projeto } \\
\hline RE Processo & den & nda & viab & dade & $\overline{\bmod }$ & gico & $\bmod$ & ísico & cons & ução & impl & tação \\
\hline REQUISITOS & FR & NFR & FR & NFR & FR & NFR & FR & NFR & FR & NFR & FR & NFR \\
\hline descobrimento & 1 & 1 & 2 & 2 & 3 & 3 & 4 & 4 & & & & \\
\hline análise & & & $\mathrm{X}$ & & $\mathrm{X}$ & $\mathrm{X}$ & $\mathrm{X}$ & $\mathrm{X}$ & & & & \\
\hline validação & & & $\mathrm{X}$ & & $\mathrm{X}$ & $\mathrm{X}$ & $\mathrm{X}$ & $\mathrm{X}$ & & & & \\
\hline documentação & $\mathrm{X}$ & $\mathrm{X}$ & $\mathrm{X}$ & $\mathrm{X}$ & $\mathrm{X}$ & $\mathrm{X}$ & $\mathrm{X}$ & $\mathrm{x}$ & $\mathrm{X}$ & $\mathrm{X}$ & & \\
\hline gerência & & & & & & & & & $\mathrm{x}$ & $\mathrm{X}$ & $\mathrm{X}$ & $\mathrm{x}$ \\
\hline
\end{tabular}

legenda: $\mathrm{x}=$ ocorrência de processos de requisitos $(1,2,3,4$, grau de detalhamento, do geral para específico) requisitos: $\quad \mathrm{FR}=$ requisitos funcionais (o que é e o que faz)

$\mathrm{NFR}=$ requisitos não-funcionais (qualidade do que é e do que faz)

O tratamento da informação, requisitos no ciclo de vida do produto, conforme a visão anteriormente disposta, parte da idéia de que em cada fase de projeto tem-se uma versão de descrição de requisitos, tanto funcionais (FR) como não-funcionais (NFR) [4,6], que se constituem em um produto intermediário no projeto, para cada fase de projeto. Isto não significa que as fases são como blocos fechados, mas que é necessário obter uma versão negociada do produto em cada fase e, a cada passagem de fase, este produto se consolida com refinamentos sucessivos, agregando informações também à fase anterior.

A informação é um produto que envolve opinião e ponto de vista de pessoas $[13,14]$, com interesses e prioridades diversos. Para captura, são utilizadas técnicas apropriadas ao ambiente e adequadas ao perfil e disponibilidade de tempo do público-alvo. Para representação, são utilizadas ferramentas de domínio do engenheiro de requisitos [5,8] que, necessariamente, não são de conhecimento e domínio da fonte de informação.

O planejamento, a execução, o controle e a avaliação de resultados são essenciais na aplicação dos processos da Engenharia de Requisitos em um projeto:

- o descobrimento de requisitos, que inicia na fase de entendimento da demanda e somente pode-se dar por concluído ao final da fase de modelo físico da solução de projeto. Isto se justifica porque é um processo indutivo e é completado à medida que a informação é discutida detalhadamente;

- a análise de requisitos, que se faz presente na fase de estudos de viabilidade, objetiva a verificação de conflitos de informação e é mais aprofundado com as informações das fases de modelo conceitual e modelo físico do projeto. Trata as relações de dependência e de relacionamento entre os requisitos;

- a validação de requisitos é complementar ao processo de análise de requisitos. Trata as relações de exigências, do ponto de vista dos stakeholder, como fonte para qualificação de prioridade de implementação e precedência dos requisitos; 
- a documentação, que se constitui em uma atividade contínua de registro das informações obtidas da fase de entendimento da demanda até a implantação, incluindo peculiaridades da fase de construção;

- o processo de gestão de mudança de requisitos [5], justifica-se em relação à versão final negociada de requisitos, após a fase de modelo físico, ao iniciar a fase de construção. Entretanto, o rastreamento de requisitos [15] quanto à relação de dependência, prioridade, precedência de informação, desde que se faça uso de uma ferramenta adequada de registro destes atributos em uma base de dados específica, é possível tratar durante todo o processo de documentação.

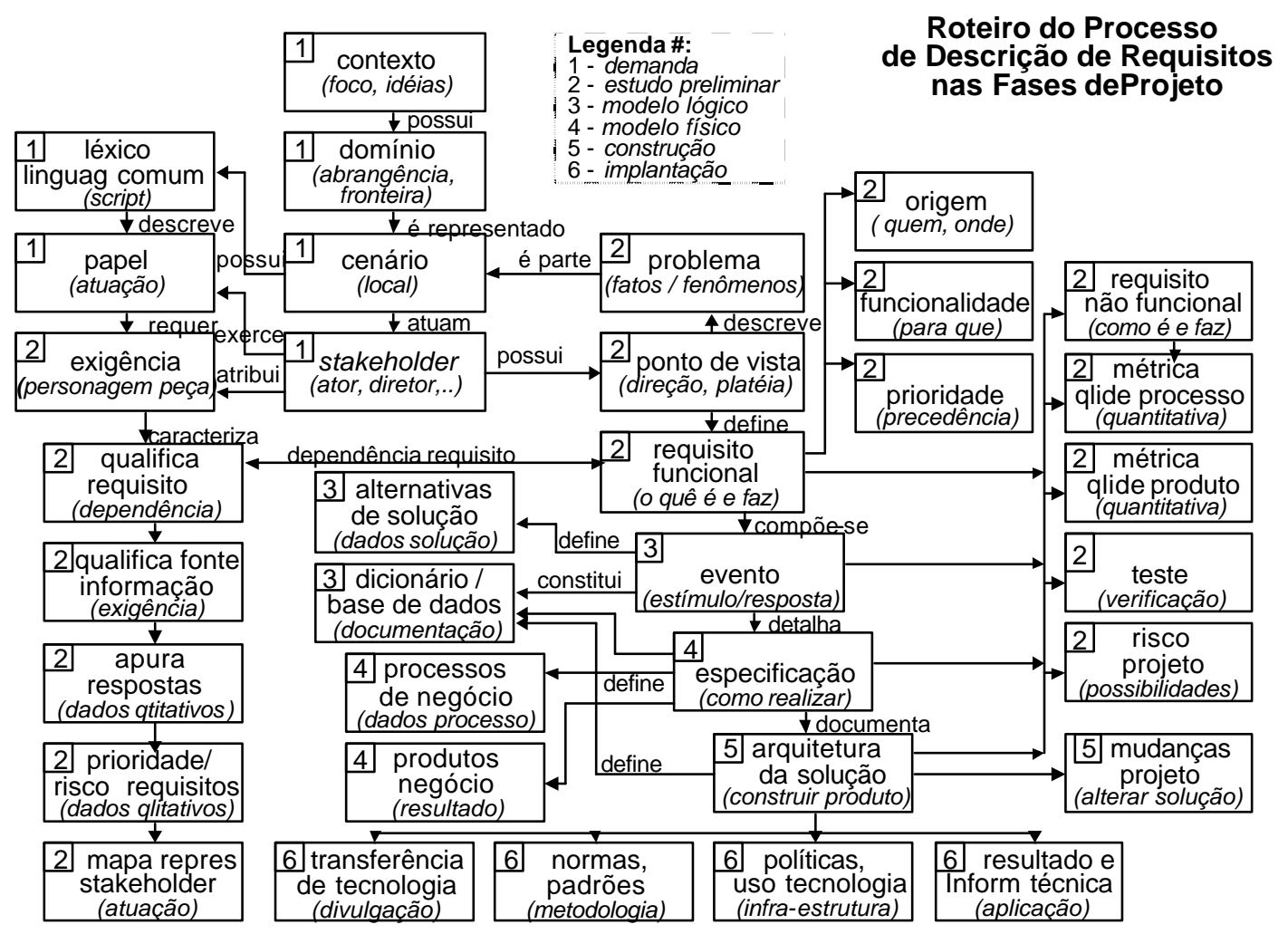

Figura.2 - Roteiro de Descrição de Requisitos, nas Fases de Projeto

A execução sistemática dos processos depende da aplicação de um roteiro (script), conforme apresentado na figura.2, direciona a constituição do produto referenciado pelos requisitos, considerando-se as fases de projeto da proposta. O roteiro constitui-se de duas etapas fundamentais: a primeira, abrange as fases 1 e 2 de projeto, respectivamente, demanda e estudo de viabilidade; a segunda abrange as fases de modelo lógico, físico, construção e implantação do projeto. A primeira fase trata do conhecimento e priorização dos requisitos; a segunda fase trata do refinamento dos requisitos, propiciado pelo detalhamento dos modelos e implementação de alternativa de solução selecionada.

\section{Modelo para Certificação}

Para a aplicação do modelo de abordagem, foi feita uma adaptação do guia de referência de Sommerville [18], ao roteiro do processo de descrição de requisitos (figura.2), revisada após a aplicação prática em projetos com escopos diferentes. 
O guia de referência, em cada fase de projeto, é apresentado em quatro partes: lista de atividades, processos da Engenharia de Requisitos, técnicas de captura e de representação dos requisitos e operacionalização da avaliação do resultado.

O foco do modelo é com o resultado, de forma que o produto é o componente principal. Cada fase de projeto é atendida por processos da Engenharia de Requisitos, constituídos de atividades, utilizando técnicas para geração de produtos. A execução das fases de projeto é direcionada pelos requisitos definidos como prioritários. Cada fase de projeto deve ter seu planejamento, sua execução e controle e a avaliação de seus produtos, privilegiando a qualidade do processo e, conseqüentemente, a qualidade do produto final.

A etapa de planejamento faz uso das atividades aplicáveis ao projeto, para os produtos esperados. O uso do roteiro (script) apóia o fluxo de procedimentos e, a aplicação do guia (checklist) adequado ao projeto, permite a avaliação do processo e dos produtos resultantes.

As etapas de execução e de controle caracterizam-se pelo desenvolvimento dos produtos da fase, realizando as atividades previstas para a elaboração dos mesmos.

A etapa de avaliação verifica a condição de uso (padrão ou convencional) da atividade para o projeto e a checagem (obrigatória ou opcional) do resultado.

\subsection{Fase de Entendimento da Demanda}

$\mathrm{Na}$ fase de entendimento da demanda inicial (tabela.2), obtém-se os requisitos como informações genéricas, não declaradas, serão baseadas no entendimento do contexto do negócio [3,13], do ponto de vista dos stakeholder envolvidos com a encomenda do produto.

Tabela.2 - Guia de Referência da Fase de Entendimento de Demanda

\begin{tabular}{|c|c|c|c|c|c|c|c|c|c|}
\hline guia de referência - entendimento demanda & \multicolumn{5}{|c|}{ (*) processo } & técnica & \multicolumn{3}{|c|}{ (\#) operacionalização } \\
\hline atividades da fase de projeto & des & ana & val & doc & ger & captura/repres & interpretação & uso & ckl \\
\hline definir o contexto & $\mathrm{x}$ & & & $\mathrm{x}$ & & conhecimento & objeto de estudo & $\mathrm{p}$ & o \\
\hline conhecer a linguagem comum ao contexto & $\mathrm{x}$ & & & $\mathrm{x}$ & & conhecimento & forma de comunicação & $\mathrm{p}$ & $\mathrm{o}$ \\
\hline conhecer a documentação histórica & $\mathrm{x}$ & & & $\mathrm{x}$ & & conhecimento & o que existe & $\mathrm{p}$ & $\mathrm{o}$ \\
\hline observar as restrições de domínio & $\mathrm{x}$ & & & $\mathrm{x}$ & & conhecimento & fronteiras & $\mathrm{p}$ & o \\
\hline definir fronteiras do sistema & $\mathrm{x}$ & & & $\mathrm{x}$ & & conhecimento & domínio aplicação & $\mathrm{p}$ & 0 \\
\hline identificar cenários para descobrir requisitos & $\mathrm{x}$ & & & $\mathrm{x}$ & & conhecimento & ambiente & $\mathrm{p}$ & $\mathrm{o}$ \\
\hline identificar os stakeholder (internos e externos) & $\mathrm{x}$ & & & $\mathrm{x}$ & & conhecimento & universo stakeholder & $\mathrm{p}$ & $\mathrm{o}$ \\
\hline identificar papéis/responsabilidades stakeholder & $\mathrm{x}$ & & & $\mathrm{x}$ & & conhecimento & quem é e o que faz & $\mathrm{p}$ & $\mathrm{o}$ \\
\hline ser sensível a fatores políticos e organizacionais & $\mathrm{x}$ & & & $\mathrm{x}$ & & conhecimento & fonte de influências & $\mathrm{c}$ & $\mathrm{x}$ \\
\hline usar conceitos de negócio no descobrimento & $\mathrm{x}$ & & & $\mathrm{x}$ & & conhecimento & negócio & $\mathrm{c}$ & $\mathrm{x}$ \\
\hline estabelecer um patrocinador para o projeto & $\mathrm{x}$ & & & $\mathrm{x}$ & & conhecimento & responsável contrato & $\mathrm{c}$ & o \\
\hline assegurar a participação dos stakeholder & $\mathrm{x}$ & & & $\mathrm{x}$ & & conhecimento & compromisso & $\mathrm{c}$ & $\mathrm{o}$ \\
\hline gerar documento inicial de demanda & $\mathrm{x}$ & & & $\mathrm{x}$ & & conhecimento & documento demanda & $\mathrm{p}$ & $\mathrm{o}$ \\
\hline
\end{tabular}

As atividades devem seguir o roteiro para contextualização do assunto.

Os processos desta fase abrangem o descobrimento e a documentação do que é para ser feito e para quem é. As técnicas utilizadas para captura devem ser as adequadas ao ambiente do negócio, observando a disponibilidade de tempo da fonte de informação. A representação da informação é documental, podendo ser em linguagem natural.

O produto é um documento inicial de demanda, cujo conteúdo orienta-se pelos elementos constitutivos do script (figura.2, \#1): contexto, domínio da aplicação, cenários, linguagem comum ao contexto, stakeholder, papéis e responsabilidades dos stakeholder, cuja finalidade é orientar o planejamento das atividades da fase seguinte, o estudo de viabilidade. 
A certificação de qualidade desta fase está em cumprir os itens obrigatórios e o foco principal é o entendimento do contexto do negócio e identificar o universo dos stakeholder.

\subsection{Fase de Estudo de Viabilidade (estudo preliminar)}

$\mathrm{Na}$ fase de estudo de viabilidade (tabela.3), o conhecimento depende da participação de pessoas do cliente e com a habilidade para tal, necessitando de representação dos níveis decisórios da organização.

Tabela.3 - Guia de Referência da Fase de Estudo de Viabilidade

\begin{tabular}{|c|c|c|c|c|c|c|c|c|c|}
\hline guia de referência - estudo de viabilidade & \multicolumn{5}{|c|}{$(*)$ processo } & \multirow{2}{*}{\begin{tabular}{|c|} 
técnica \\
captura/repres \\
\end{tabular}} & \multicolumn{3}{|c|}{ (\#) operacionalização } \\
\hline atividades da fase de projeto & des & ana & val & doc & ger & & interpretação & uso & ckl \\
\hline usar templates para descrição de requisitos & $\mathrm{x}$ & & & $\mathrm{x}$ & & descrição & roteiro descrição & $\mathrm{c}$ & $\mathrm{x}$ \\
\hline usar linguagem simples e concisa & $\mathrm{x}$ & & & $\mathrm{x}$ & & descrição & sentença simples & $\mathrm{p}$ & $\mathrm{o}$ \\
\hline suplementar linguagem natural c/outra descrição & $\mathrm{x}$ & & & $\mathrm{x}$ & & descrição & notações, fórmulas & $\mathrm{c}$ & $\mathrm{x}$ \\
\hline identificar o problema/oportunidade de solução & $\mathrm{x}$ & & & $\mathrm{x}$ & & conhecimento & denominação & $\mathrm{p}$ & $\mathrm{o}$ \\
\hline descrever requisitos funcionais & $\mathrm{x}$ & & & $\mathrm{x}$ & & conhecimento & essência & $\mathrm{p}$ & $\mathrm{o}$ \\
\hline identificar requisitos não-funcionais & $\mathrm{x}$ & & & $\mathrm{x}$ & & conhecimento & essência & $\mathrm{c}$ & $\mathrm{x}$ \\
\hline identificar restrições, preferências e premissas & $\mathrm{x}$ & & & $\mathrm{x}$ & & conhecimento & essência & $\mathrm{c}$ & $\mathrm{x}$ \\
\hline especificar requisitos quantitativamente & $\mathrm{x}$ & & & $\mathrm{x}$ & & conhecimento & volume & $\mathrm{c}$ & $\mathrm{x}$ \\
\hline coletar requisitos de múltiplos pontos de vista & $\mathrm{x}$ & & & $\mathrm{x}$ & & conhecimento & diversidade pontovista & $\mathrm{p}$ & o \\
\hline associar papéis e responsabilidades stakeholder & $\mathrm{x}$ & & & $\mathrm{x}$ & & conhecimento & quem é e o que faz & $\mathrm{p}$ & $\mathrm{o}$ \\
\hline capturar riscos do projeto & $\mathrm{x}$ & & & $\mathrm{x}$ & & conhecimento & técnica & $\mathrm{c}$ & $\mathrm{x}$ \\
\hline capturar métricas preliminares de qualidade & $\mathrm{x}$ & & & $\mathrm{x}$ & & conhecimento & técnica & $\mathrm{c}$ & $\mathrm{x}$ \\
\hline capturar marcos de testes do projeto & $\mathrm{x}$ & & & $\mathrm{x}$ & & conhecimento & técnica & $\mathrm{c}$ & $\mathrm{x}$ \\
\hline fazer reuso de requisitos & & $\mathrm{x}$ & & $\mathrm{x}$ & & análise & economia trabalho & $\mathrm{c}$ & $\mathrm{x}$ \\
\hline identificar unicamente cada requisito & & $\mathrm{x}$ & & $\mathrm{x}$ & & análise & denominação & $\bar{p}$ & $\mathrm{o}$ \\
\hline identificar a funcionalidade do requisito & & $\mathrm{x}$ & & $\mathrm{x}$ & & análise & denominação & $\mathrm{p}$ & $\mathrm{o}$ \\
\hline identificar a relação de dependência requisito & & $\mathrm{x}$ & & $\mathrm{x}$ & & análise & denominação & $\mathrm{p}$ & o \\
\hline qualificar a exigência do requisito & & $\mathrm{x}$ & & $\mathrm{x}$ & & análise & denominação & $\mathrm{p}$ & $\mathrm{O}$ \\
\hline usar checklist para análise dos requi & & $\mathrm{x}$ & & $\mathrm{x}$ & & análise & analisar requisitos & $\mathrm{p}$ & $\mathrm{o}$ \\
\hline classificar requisitos c/abordagem multidimensão & & $\mathrm{x}$ & & $\mathrm{x}$ & & análise & relacionamentos & $\mathrm{c}$ & $\mathrm{x}$ \\
\hline complementar a linguagem comum do contexto & & $\mathrm{x}$ & & $\mathrm{x}$ & & análise & forma de comunicação & $\mathrm{c}$ & $\mathrm{x}$ \\
\hline usar plano para conflitos e resolução conflitos & & $\mathrm{x}$ & & $\mathrm{x}$ & & negociação & relacionamento & $\mathrm{c}$ & $\mathrm{x}$ \\
\hline $\begin{array}{l}\text { usar matrizes de interação para encontrar conflitos } \\
\text { e sobreposição de pontos de vista de requisitos }\end{array}$ & & $\mathrm{x}$ & & $\mathrm{x}$ & & negociação & relacionamentos & $\mathrm{c}$ & o \\
\hline prover software para suporte a negociações & & $\mathrm{x}$ & & $\mathrm{x}$ & & negociação & registro aplicação & $\mathrm{c}$ & $\mathrm{x}$ \\
\hline usar checklist de validação & & & $\mathrm{x}$ & $\mathrm{x}$ & & validação & atributos críticos & $\mathrm{p}$ & o \\
\hline usar equipes multidisciplinares para revisão & & & $\mathrm{x}$ & $\mathrm{x}$ & & validação & representa stakeholder & $\mathrm{p}$ & $\mathrm{o}$ \\
\hline analisar representatividade stakeholder na revisão & & & $\mathrm{x}$ & $\mathrm{x}$ & & validação & representa stakeholder & $\mathrm{c}$ & $\mathrm{o}$ \\
\hline priorizar requisitos funcionais & & & $\mathrm{x}$ & $\mathrm{x}$ & & validação & priorização & $\mathrm{p}$ & $\mathrm{O}$ \\
\hline avaliar viabilidade do sistema & & & $\mathrm{x}$ & $\mathrm{x}$ & & validação & realizar estudo prévio & $\mathrm{p}$ & $\mathrm{o}$ \\
\hline gerar documento linguagem comum ao contexto & & & & $\mathrm{x}$ & & documentação & documento & $\mathrm{p}$ & $\mathrm{o}$ \\
\hline $\begin{array}{l}\text { gerar documento de requisitos funcionais } \\
\text { qualificados e priorizados e, req.não-funcionais }\end{array}$ & & & & $\mathrm{x}$ & & documentação & documento & $\mathrm{p}$ & 0 \\
\hline gerar documento risco implementação requisitos & & & & $\mathrm{X}$ & & documentação & documento & $\mathrm{p}$ & $\mathrm{o}$ \\
\hline gerar documento de rastreabilidade de requisitos & & & & $\mathrm{x}$ & & documentação & documento & $\mathrm{p}$ & $\mathrm{o}$ \\
\hline
\end{tabular}

As atividades desta fase são as mais extensas, abrangem quatro processos para requisitos e são fundamentais para o conhecimento da informação. Os processos são iterativos, constituem-se de descobrimento, análise, validação e documentação.

$\mathrm{O}$ processo de descobrimento deve ser estimulado na linguagem em que a fonte de informação está acostumada, deve existir negociação prévia do método a ser aplicado e o compromisso a ser assumido. Deve-se ter o cuidado com palavras rejeitadas no contexto, por 
exemplo, problema. O resultado da caracterização do requisito depende da interpretação do engenheiro de requisitos, pois a metodologia a ser aplicada no processo deve ser menos formal, por exemplo, distinção entre requisito funcional (função) e não-funcional (qualidade da função). As informações deverão ser obtidas de pessoas com pontos de vista variados, segundo Leite [14], dentro de um contexto definido.

O processo de análise deve ser aplicado aos requisitos funcionais para avaliar conflitos [1,2], ambigüidades, repetição. $O$ de validação, deve ser aplicado aos requisitos funcionais para verificar a relação de dependência, precedência e prioridade de atendimento e, por fim, o processo de documentação deve registrar e representar toda a informação coletada e analisada. A representação da informação é documental, podendo ser em linguagem natural, complementada com alguns modelos de representação, de domínio do analista.

Os produtos são: documento da linguagem comum ao contexto, documento descritivo dos requisitos funcionais qualificados e priorizados e a visão inicial de requisitos nãofuncionais ou de qualidade; documento preliminar de riscos de implantação de requisitos; documento que visualiza o rastreamento de requisitos, condições preliminares para teste e características genéricas de qualidade. Os conteúdos dos documentos orientam-se pelos elementos constitutivos do script (figura.2, \#2): fundamentados a partir do ponto de vista do problema e do requisito. A finalidade é orientar o planejamento das atividades da fase seguinte, a construção do modelo lógico com definição de alternativas de solução.

A certificação de qualidade desta fase está em cumprir as atividades obrigatórias e o foco principal é a obtenção da descrição dos requisitos representativos do contexto, devidamente qualificados, relacionados e priorizados, envolvendo uma amostra representativa do universo dos stakeholder.

\subsection{Fase de Modelo Lógico}

$\mathrm{Na}$ fase de modelo lógico (tabela.4), o processo de descobrimento (tabela.1) apresenta o esforço na definição clara de papéis e de responsabilidades dos stakeholder pelos eventos, a visão preliminar dos processos de negócio, restrições e premissas.

Tabela.4 - Guia de Referência da Fase de Modelo Lógico

\begin{tabular}{|c|c|c|c|c|c|c|c|c|c|}
\hline guia de referência - modelo lógico & \multicolumn{5}{|c|}{ (*) processo } & técnica & \multicolumn{3}{|c|}{ (\#) operacionalização } \\
\hline atividades da fase de projeto & des & ana & val & doc & ger & captura/repres & interpretação & uso & ckl \\
\hline associar papéis stakeholder aos eventos & $\mathrm{x}$ & & & $\mathrm{x}$ & & conhecimento & quem é e o que faz & $\mathrm{p}$ & $\mathrm{o}$ \\
\hline complementar a linguagem comum do contexto & $\mathrm{x}$ & & & $\mathrm{x}$ & & conhecimento & forma de comunicação & $\mathrm{c}$ & $\mathrm{x}$ \\
\hline capturar riscos do projeto & $\mathrm{x}$ & & & $\mathrm{x}$ & & conhecimento & técnica & $\mathrm{c}$ & $\mathrm{x}$ \\
\hline capturar marcos de teste & $\mathrm{x}$ & & & $\mathrm{x}$ & & conhecimento & técnica & $\mathrm{c}$ & $\mathrm{x}$ \\
\hline capturar métricas de qualidade & $\mathrm{x}$ & & & $\mathrm{x}$ & & conhecimento & técnica & $\mathrm{c}$ & $\mathrm{x}$ \\
\hline usar diagramas apropriados & $\mathrm{x}$ & & & $\mathrm{x}$ & & modelagem & representação & $\mathrm{c}$ & $\mathrm{x}$ \\
\hline usar métodos para modelagem sistemas & $\mathrm{x}$ & & & $\mathrm{x}$ & & modelagem & método & $\mathrm{p}$ & $\mathrm{o}$ \\
\hline representar os eventos com visão dos processos & $\mathrm{x}$ & & & $\mathrm{x}$ & & modelagem & representação & $\mathrm{p}$ & $\mathrm{o}$ \\
\hline incrementar o dicionário de dados & $\mathrm{x}$ & & & $\mathrm{x}$ & & modelagem & representação dado & $\mathrm{p}$ & $\mathrm{o}$ \\
\hline documentar os links entre requisitos e modelos & $\mathrm{x}$ & & & $\mathrm{x}$ & & modelagem & refinamento & $\mathrm{p}$ & $\mathrm{o}$ \\
\hline desenvolver modelos complementares sistema & $\mathrm{x}$ & & & $\mathrm{x}$ & & modelagem & ilustrações & $\mathrm{c}$ & $\mathrm{x}$ \\
\hline checar requisitos não-funcionais, qualidade & & $\mathrm{x}$ & & $\mathrm{x}$ & & Análise & essência & $\mathrm{c}$ & $\mathrm{O}$ \\
\hline definir alternativas de solução & & $\mathrm{x}$ & & $\mathrm{x}$ & & Análise & representação solução & $\mathrm{p}$ & $\mathrm{o}$ \\
\hline proceder refinamento da descrição dos requisitos & & & $\mathrm{x}$ & $\mathrm{x}$ & & validação & complementar dados & $\mathrm{c}$ & $\mathrm{x}$ \\
\hline gerar documento com eventos/processos & & & & $\mathrm{x}$ & & documentação & documento & $\mathrm{p}$ & o \\
\hline gerar documento versão base de dados & & & & $\mathrm{x}$ & & documentação & documento & $\mathrm{p}$ & $\mathrm{o}$ \\
\hline \begin{tabular}{|l} 
gerar documento de alternativas de solu \\
\end{tabular} & & & & $\mathrm{x}$ & & documentação & documento & $\mathrm{p}$ & $\mathrm{o}$ \\
\hline
\end{tabular}

(*) processo: des - descobrimento, ana - análise, val - validação, doc - documentação, ger - gerência

(\#) operacionalização uso: p-padrão, c-convencional 
As atividades desta fase são específicas de detalhamento dos eventos do negócio e, conseqüentemente, contribuem para o refinamento dos requisitos já identificados.

Os processos são iterativos, constituem-se de descobrimento, análise, validação e de documentação dos eventos associados aos requisitos priorizados. Os processos de análise e de validação devem ser aplicados aos requisitos funcionais e não-funcionais, com o objetivo de complementar as informações existentes. O processo de documentação deve registrar e representar toda a informação coletada e analisada. As técnicas utilizadas para captura e representação devem ser adequadas a uma linguagem acessível ao público-alvo, observando a essência do processo que é a comunicação entre os participantes. A representação da informação é documental, utilizando-se modelos.

Os produtos são: documento do modelo de representação dos eventos associados aos requisitos com papéis e responsabilidades dos stakeholder, documento descritivo da base de dados, documento preliminar de riscos e de teste de projeto, métricas de qualidade e do documento que propõe alternativas de solução. Os conteúdos dos documentos orientam-se pelos elementos constitutivos do script (figura.2, \#3): detalhando os eventos a partir dos requisitos funcionais, gerando opções de alternativas de solução, cuja finalidade é orientar o planejamento das atividades da fase seguinte, a construção do modelo físico da alternativa selecionada para a especificação técnica de processos e produtos do negócio.

A certificação de qualidade desta fase está em cumprir as atividades obrigatórias e o foco principal é a obtenção dos modelos de representação dos eventos, especificando alternativas de solução com o uso de tecnologia da informação.

\subsection{Fase de Modelo Físico}

A fase de modelo físico (tabela.5), representa na especificação da solução, os processos de negócio e os produtos.

Tabela.5 - Guia de Referência da Fase de Modelo Físico

\begin{tabular}{|c|c|c|c|c|c|c|c|c|c|}
\hline guia de referência - modelo físico & \multicolumn{5}{|c|}{ (*) processo } & técnica & \multicolumn{3}{|c|}{ (\#) operacionalização } \\
\hline atividades da fase de projeto & des & ana & val & doc & ger & captura/repres & interpretação & uso & ckl \\
\hline capturar riscos do projeto & $\mathrm{X}$ & & & $\mathrm{X}$ & & conhecimento & técnica & $\mathrm{p}$ & $\mathrm{O}$ \\
\hline capturar marcos de teste & $\mathrm{X}$ & & & $\mathrm{x}$ & & conhecimento & técnica & $\mathrm{p}$ & $\mathrm{O}$ \\
\hline capturar métricas de qualidade & $\mathrm{X}$ & & & $\mathrm{x}$ & & conhecimento & técnica & $\mathrm{p}$ & $\mathrm{O}$ \\
\hline definir sistemas usando especificação formal & $\mathrm{X}$ & & & $\mathrm{x}$ & & descrição & notações linguagem & $\mathrm{c}$ & $\mathrm{X}$ \\
\hline modelar a arquitetura do sistema & $\mathrm{X}$ & & & $\mathrm{X}$ & & modelagem & link subsistemas & $\mathrm{p}$ & o \\
\hline definir processo operacional & $\mathrm{X}$ & & & $\mathrm{X}$ & & modelagem & fácil de executar & $\mathrm{p}$ & $\mathrm{O}$ \\
\hline representar os processos de negócio & $\mathrm{X}$ & & & $\mathrm{x}$ & & modelagem & representação & $\mathrm{p}$ & $\mathrm{O}$ \\
\hline representar os produtos de negócio & $\mathrm{X}$ & & & $\mathrm{X}$ & & modelagem & representação & $\mathrm{p}$ & $\mathrm{O}$ \\
\hline estabelecer o modelo físico de dados & $\mathrm{X}$ & & & $\mathrm{X}$ & & modelagem & representação dado & $\mathrm{p}$ & $\mathrm{O}$ \\
\hline priorizar requisitos não-funcionais & & $\mathrm{X}$ & & $\mathrm{x}$ & & análise & essência / prioridade & $\mathrm{p}$ & $\mathrm{O}$ \\
\hline proceder refinamento da descrição dos requisitos & & & $\mathrm{x}$ & $\mathrm{X}$ & & validação & complementar dados & c & $\mathrm{X}$ \\
\hline definir políticas de gestão de requisitos & & & & $\mathrm{x}$ & $\mathrm{x}$ & gerência & mudança & $\mathrm{p}$ & $\mathrm{O}$ \\
\hline definir políticas de gestão de qualidade & & & & $\mathrm{x}$ & $\mathrm{x}$ & gerência & métricas qualidade & $\mathrm{p}$ & $\mathrm{O}$ \\
\hline definir políticas de rastreamento de requisitos & & & & $\mathrm{x}$ & $\mathrm{x}$ & gerência & dependência requisitos & $\mathrm{c}$ & $\mathrm{X}$ \\
\hline manter condição de rastreamento manual & & & & $\mathrm{x}$ & $\mathrm{x}$ & gerência & rastreamento & $\mathrm{c}$ & $\mathrm{X}$ \\
\hline gerar documento de processos e produtos & & & & $\mathrm{X}$ & & documentação & documento & $\mathrm{p}$ & $\mathrm{O}$ \\
\hline gerar documento versão base de dados & & & & $\mathrm{x}$ & & documentação & documento & $\mathrm{p}$ & $\mathrm{O}$ \\
\hline gerar documento de gerência de riscos & & & & $\mathrm{x}$ & & documentação & documento & $\mathrm{p}$ & o \\
\hline gerar documento de gerência de testes & & & & $\mathrm{x}$ & & documentação & documento & $\mathrm{p}$ & $\mathrm{O}$ \\
\hline gerar documento de métricas de qualidade & & & & $\mathrm{X}$ & & documentação & documento & $\mathrm{p}$ & $\mathrm{O}$ \\
\hline
\end{tabular}

(*) processo: des - descobrimento, ana - análise, val - validação, doc - documentação, ger - gerência

(\#) operacionalização uso: p-padrão, c-convencional ckl: o-obrigatório, x-opcional 
As atividades desta fase são específicas de detalhamento das funções de solução. É essencial completar a descrição e o refinamento dos requisitos não-funcionais, as características de qualidade e as métricas, principalmente, a definição de prioridade de atendimento dos mesmos em relação às necessidades de negócio e à solução de software adotada.

Os processos são iterativos, constituem-se de descobrimento, análise, validação e documentação da especificação de solução em como atender aos requisitos estabelecidos. As técnicas utilizadas para captura e representação são de domínio da equipe de projeto e voltadas para o público-alvo que construirá o projeto. A representação da informação é documental, utilizando-se modelos.

Os produtos são: documento do modelo de representação dos processos e produtos de negócio, documento descritivo da base de dados, documento de riscos de projeto, documento de testes, documento que propõe métricas para avaliação de qualidade do produto de software. Os conteúdos dos documentos orientam-se pelos elementos constitutivos do script (figura.2, \#4): detalhando os processos e produtos a partir da especificação da solução, orientando a arquitetura e comunicação entre as funções, cuja finalidade é orientar o planejamento das atividades da fase seguinte, a construção do software e, especialmente, fundamentar os processos de gerência de requisitos, gerência de testes, gerência de riscos e gerência de qualidade do software.

\subsection{Fase de Construção}

A fase de construção (tabela.6) além de representar a arquitetura das funções de software, é o marco inicial dos processos de gestão de requisitos, de riscos, de testes e de qualidade, pois é nesta fase que se tem a descrição completa da arquitetura da função que implementará a funcionalidade e as características de qualidade dos requisitos estabelecidos. Um fato particular é o registro das solicitações de mudanças que ocorrerem após o projeto da solução ter sido concluído.

Tabela.6 - Guia de Referência da Fase de Construção

\begin{tabular}{|c|c|c|c|c|c|c|c|c|c|}
\hline guia de referência - construção & \multicolumn{5}{|c|}{ (*) processo } & técnica & \multicolumn{3}{|c|}{ (\#) operacionalização } \\
\hline atividades da fase de projeto & des & ana & val & doc & ger & captura/repres & interpretação & uso & ckl \\
\hline detalhar a arquitetura das funções & $\mathrm{x}$ & & & $\mathrm{x}$ & & modelagem & link subsistemas & $\mathrm{p}$ & o \\
\hline checar requisitos não-funcionais & & $\mathrm{X}$ & & $\mathrm{x}$ & & análise & priorização & $\mathrm{p}$ & $\mathrm{o}$ \\
\hline avaliação de riscos de requisitos & & $\mathrm{x}$ & & $\mathrm{x}$ & & análise & prioridade, qualidade & $\mathrm{p}$ & $\mathrm{o}$ \\
\hline propor casos de teste de requisitos & & & $\mathrm{x}$ & $\mathrm{x}$ & & validação & teste qualidade & $\mathrm{p}$ & o \\
\hline organizar inspeção formal de requisitos & & & $\mathrm{x}$ & $\mathrm{x}$ & & validação & checagem requisitos & $\mathrm{c}$ & $\mathrm{x}$ \\
\hline identificar mudanças na solução projetada & & & $\mathrm{x}$ & $\mathrm{x}$ & & validação & mudança de requisitos & $\mathrm{c}$ & $\mathrm{x}$ \\
\hline gerar documento de arquitetura de funções & & & & $\mathrm{x}$ & & documentação & documento & $\mathrm{p}$ & $\mathrm{o}$ \\
\hline gerar documento de comunicação entre funções & & & & $\mathrm{x}$ & & documentação & documento & $\mathrm{p}$ & $\mathrm{o}$ \\
\hline gerar documento versão base de dados & & & & $\mathrm{x}$ & & documentação & documento & $\mathrm{p}$ & $\mathrm{o}$ \\
\hline gerar documento de gerência de riscos & & & & $\mathrm{x}$ & & documentação & documento & $\mathrm{p}$ & $\mathrm{o}$ \\
\hline gerar documento de gerência de testes & & & & $\mathrm{x}$ & & documentação & documento & $\mathrm{p}$ & $\mathrm{o}$ \\
\hline gerar documento de métricas de qualidade & & & & $\mathrm{x}$ & & documentação & documento & $\mathrm{p}$ & $\mathrm{o}$ \\
\hline
\end{tabular}
(\#) processo:
(\#) operacionalização
ckl: o - obrigatório, x - opcional
ckloc - documentac̃ão, ger - gerência

As atividades desta fase correspondem ao registro das ocorrências de construção do software. Os processos são iterativos, constituem-se de descobrimento, análise, validação e de documentação da arquitetura e comunicação entre funções em como atender aos requisitos. 
As técnicas utilizadas para captura e representação são de domínio da equipe que desenvolve o projeto. A representação da informação é documental.

Os produtos são: documento do modelo de representação da arquitetura e comunicação entre funções, documento descritivo da base de dados, documento de riscos de projeto, documento de validação de testes, documento que valida métricas para avaliação de qualidade do software. Os conteúdos dos documentos orientam-se pelos elementos constitutivos do script (figura.2, \#5): detalhando as funções, cuja finalidade é orientar o planejamento das atividades da fase seguinte, a implantação do software e, especialmente, fundamentar os processos de transferência de tecnologia, políticas, normas e padrões e resultados da aplicação.

A certificação de qualidade desta fase está em cumprir as atividades obrigatórias e o foco principal é o relato de avaliação dos processos de gerência de requisitos quanto às mudanças, de gerência de riscos do projeto, de casos de testes e de métricas de qualidade dos processos e dos produtos.

\subsection{Fase de Implantação}

A fase de implantação (tabela.7) é o marco inicial da divulgação da tecnologia construída, pois é nesta fase que se tem a visão completa da funcionalidade e as características de qualidade do software.

Tabela.7 - Guia de Referência da Fase de Implantação

\begin{tabular}{|c|c|c|c|c|c|c|c|c|}
\hline guia de referência - implantação & \multicolumn{4}{|c|}{$(*)$ processo } & técnica & \multicolumn{3}{|c|}{ (\#) operacionalização } \\
\hline atividades da fase de projeto & des ana & val & doc & ger & captura/repres & interpretação & uso & ckl \\
\hline escrever um esboço de manual de usuário & & & $\mathrm{x}$ & & descrição & negócio & $\mathrm{c}$ & $\bar{x}$ \\
\hline \begin{tabular}{|l|} 
definir uma estrutura padrão de documento \\
\end{tabular} & & & $\mathrm{x}$ & & descrição & formato padrão & $\mathrm{p}$ & o \\
\hline explicar como usar o documento & & & $\mathrm{x}$ & & descrição & por tipo de leitor & $\mathrm{p}$ & $\mathrm{o}$ \\
\hline fazer um relato de negócio para o sistema & & & $\mathrm{x}$ & & descrição & objetivos negócio & $\mathrm{c}$ & $\mathrm{x}$ \\
\hline definir termos especialistas & & & $\mathrm{x}$ & & descrição & glossário termos & $\mathrm{p}$ & $\mathrm{O}$ \\
\hline lay-out documento para facilidade de leitura & & & $\mathrm{x}$ & & descrição & texto fácil de ler & $\mathrm{c}$ & o \\
\hline ajuda para leitores encontrarem a informação & & & $\mathrm{x}$ & & descrição & lista conteúdo & $\mathrm{c}$ & $\mathrm{O}$ \\
\hline fazer um documento fácil para mudar & & & $\mathrm{x}$ & & descrição & fácil modificação & $\mathrm{c}$ & $\mathrm{o}$ \\
\hline usar base de dados para gerenciar requisitos & & & $\mathrm{x}$ & $\mathrm{x}$ & gerência & automatizar & $\mathrm{c}$ & $\mathrm{x}$ \\
\hline \begin{tabular}{|l|} 
definir políticas de gestão de mudanças \\
\end{tabular} & & & $\mathrm{x}$ & $\mathrm{x}$ & gerência & avaliar impacto & $\mathrm{p}$ & o \\
\hline identificar requisitos voláteis & & & $\mathrm{x}$ & $\mathrm{x}$ & gerência & negócio & $\mathrm{c}$ & $\mathrm{X}$ \\
\hline registrar requisitos rejeitados & & & $\mathrm{x}$ & $\mathrm{x}$ & gerência & algo já discutido & $\mathrm{c}$ & $\mathrm{x}$ \\
\hline gerar documento de transferência de tecnologia & & & $\mathrm{x}$ & & documentação & documento & $\mathrm{p}$ & $\mathrm{o}$ \\
\hline
\end{tabular}

O processo é essencialmente documental. As técnicas utilizadas para captura e representação dos resultados são voltadas para o usuário. A representação da informação é a formalização de procedimentos e de uso dos produtos.

Os produtos são: documento do modelo de uso da tecnologia, normas e padrões e resultados aplicativos. Os conteúdos dos documentos orientam-se pelos elementos constitutivos do script (figura.2, \#6): detalhando os procedimentos, cuja finalidade é orientar o planejamento das atividades de implantação, a gestão de mudanças e a gestão de qualidade.

A certificação de qualidade desta fase está em cumprir as atividades obrigatórias e o foco principal é o preparo do ambiente e as condições e políticas para implantação do software. 


\section{Aplicação do Modelo}

A aplicação prática relata o estudo de uma organização estatal que foi constituída para a gestão de fundo capitalizado de previdência pública, de regime próprio, com capitalização e foco atuarial. A organização, com três anos de existência, juntou atribuições de um órgão que tratava pensões, com as atribuições de outro órgão que tratava de aposentadorias e contribuições para a futura aposentadoria. Com isto, herdou toda a base de dados e sistemas dos referidos órgãos na forma em que funcionavam e teve alocados recursos humanos das mais diversas áreas de atuação previdenciária, notadamente, governo federal, estadual e municipal.

A abordagem de estudo do sistema de informações para este novo ambiente, foi mapear a situação existente e congregar esforços em andamento para a manutenção do legado e criar funcionalidades imediatas.

Utilizando-se deste material histórico, do Plano Diretor de Informática, da Legislação que constituiu a nova organização, a abordagem de encaminhamento visualizava o projeto global com fases distintas: licitação independente e, a relativa ao objeto de estudo como um todo: demanda, estudo preliminar, modelos lógico e físico, transição dos sistemas atuais e construção de nova solução.

\subsection{Fase de Entendimento da Demanda}

Tratava-se de um caso complexo de desenvolvimento de sistema de informações [23], a considerar os fatos ambientais. A demanda inicial é ter um sistema novo, um novo cadastro cujas informações só possam ser mantidas pela organização, atender ao público cliente com informações atualizadas e ter informações para gestão do negócio.

$\mathrm{O}$ problema maior em questão era como definir o que era para ser feito, quais os problemas relacionados, qual a precedência de atendimento às necessidades expostas, como os processos se inter-relacionam e, consequientemente, quais são os requisitos de funcionalidade e de qualidade dos produtos que fazem parte do contexto de demanda.

As restrições de participação de pessoal com conhecimento para serem fonte de informação dos processos era a concorrência com o desempenho diário de suas atividades, sendo estas, consideradas prioritárias.

O produto resultante desta fase foi uma proposta de trabalho contendo a análise da situação e submetendo à apreciação a metodologia de tratamento da informação a ser adotada na fase seguinte.

\subsection{Fase de Estudo de Viabilidade (preliminar)}

Após várias reuniões de negociação sobre a necessidade de comprometimento das pessoas responsáveis pela tomada decisão, ficou contratado que o trabalho pelo tamanho, volume e esforço a serem despendidos, necessitaria ser dividido em fases. Isto permitiria apresentar produtos intermediários para avaliação de resultados e negociação de continuidade

A fase de trabalho, denominada de fase de estudos preliminares ou de viabilidade, formalizou um planejamento de atividades usando a técnica de reunião presencial com representantes individuais das áreas diretivas, assessorias técnicas e gerências da organização, apresentando um cronograma, cujo objetivo era criar um produto (documento preliminar de 
requisitos) gerado com a participação do grupo decisório da organização. $\mathrm{O}$ enfoque era o conhecimento dos requisitos de negócio, priorizados.

A abordagem adotada nesta fase foi orientada pelo roteiro de descrição de requisitos (figura.2). A partir das informações sob o ponto de vista dos stakeholder, foram listados os problemas, requisitos, restrições e premissas do corpo diretivo e gerencial da organização.

$\mathrm{Na}$ seqüência, foram aplicados os processos de análise, validação e qualificação dos requisitos [19,20], obtendo-se um documento de requisitos com as informações priorizadas.

A necessidade de informação era conhecer o ambiente e a pretensão das pessoas quanto a o quê fazer para adequar à realidade da nova organização, sem deixar de atender aos processos operacionais em andamento. A documentação gerada nesta fase faz parte do conhecimento das Informações de Negócio do Sistema Previdenciário sob os aspectos: restrições de negócio, premissas visualizadas, problemas e necessidades de negócio, oportunidade de aplicação de tecnologia da informação e os requisitos do ponto de vista diretivo e gerencial, eventualmente, algumas declarações de requisitos do ponto de vista operacional.

\subsection{O Tratamento da Informação (requisitos no ciclo de vida do produto)}

O documento preliminar de requisitos resultou num total de 185 requisitos genéricos. Foi necessária uma classificação prévia pela similaridade de tratamento do tema, antes da qualificação dos requisitos para avaliação de relacionamento de dependência e respectiva prioridade definida.

Uma característica particular apareceu na negociação desta fase do trabalho. Pela complexidade e esforço a ser despendido nas atividades, principalmente pelo recurso tempo e participação das mesmas pessoas de linha de produção em repetidos processos de definição, optou-se que na fase seguinte, a de modelo lógico ou conceitual, o trabalho deveria apresentar como resultado, definição de alternativas intermediárias para implementação imediata. Alocando esforços nas atividades que inicialmente agregariam maior valor [10,17]. Para isso, deveria ser referência a hierarquia de requisitos definida na fase de estudo de viabilidade.

A negociação da fase seguinte foi condicionada a restrições e premissas:

- apresentação de proposta de alternativa de produção de resultados imediatos;

- adaptação dos sistemas existentes (inovação e melhoria);

- construção de base gerencial técnica: datawarehouse;

- atendimento da fila de solicitações em andamento;

- apresentação de alternativas de implementação de novas funções prioritárias.

\subsection{As fases Seguintes...}

A fase de modelo lógico evidenciou no ambiente da organização a necessidade de criação de uma linguagem comum (léxico), a partir da análise do vocabulário diverso existente. Outro elemento presente é necessidade da definição clara de papéis e responsabilidades para as pessoas. E, na sequiência, a formalização dos processos de negócio para a nova organização. Isto representa um esforço adicional ao processo, porque o resultado do trabalho deverá ser construir a nova forma de funcionamento organizacional.

A fase de modelo físico ficou para ser desenvolvida após a conclusão do modelo lógico e opção pelas alternativas de solução. 
Nas fases de construção e de implantação, fazia-se necessário começar pelas prioridades da organização.

\subsection{Resultados Obtidos}

O fator mais importante na aplicação da abordagem foi a evidência de que ao partir-se do modelo teórico de utilização dos processos da Engenharia de Requisitos em um projeto, deve-se sempre aproveitar o momento e as circunstâncias que levem ao conhecimento efetivo do negócio, o que é para fazer. Partindo deste princípio, foi primordial obter um documento de requisitos devidamente revisado, com as prioridades destacadas e apontando para a precedência de criação dos modelos de representação dos eventos e base de dados, na busca de alternativas de solução.

Outro fator foi ter conseguido a participação do corpo diretivo da organização, enfocando o tratamento da informação (problemas e expectativas, requisitos, restrições, premissas) e não da tecnologia. Com o mapeamento de prioridades, o corpo gerencial teve a definição das linhas de ação e apontamentos de gestão do negócio.

Do ponto de vista de certificação de qualidade, a aplicação dos processos da Engenharia de Requisitos no modelo (exceto o de gerência), foi efetiva e propiciou a referência para a negociação da seqüência do projeto.

\section{Conclusão}

A proposta de abordagem do guia de referência para contribuir no processo de certificação de qualidade em Engenharia de Requisitos é o resultado da aplicação prática em projetos com escopos diferentes, realizados até a fase de modelo físico do projeto. A observação é que o conhecimento, tendo como foco a descrição dos requisitos, leva à reflexão de alguns fatores relevantes a considerar na certificação de qualidade dos processos.

Cabe aqui relembrar a tabela.1, Relacionamento dos Processos de ER com as Fases de Projeto, como um referencial para esta análise. O processo de descrição de requisitos é uma atividade indutiva e continuada. Tem conteúdo mais genérico na fase de entendimento da demanda. À medida que se avança nas demais fases de projeto, exige-se detalhamento e mais formalismo. As descrições de funcionalidade tornam-se mais específicas e completas e as de não-funcionalidade (qualidade) também o são.

Aplicando-se a avaliação a cada término de fase de projeto, a negociação de entrega de resultados intermediários é que dirigirá a continuidade do mesmo, ou seja, a passagem para a fase seguinte do projeto ou de um novo projeto.

A capacitação e o nível de maturidade da organização devem estar voltados para a geração de produtos intermediários e/ou finais a cada fase de projeto utilizando um script formal para o conhecimento, análise, validação, documentação e gerência da informação. Da mesma forma que a utilização de um checklist para avaliação dos processos e produtos resultantes a cada fase de projeto permitirá a aceitação do produto para a fase seguinte.

Concluindo, a certificação de qualidade em Engenharia de Requisitos, compreende a somatória de fatores; a organização estar apta para aplicação dos processos e a avaliação sistemática de resultados. É um processo complexo, cujo objeto é a definição clara de requisitos e sua aplicabilidade ao ciclo de vida de um projeto. A forma de utilização dos processos é quem dirigirá a continuidade e aperfeiçoamento de procedimentos na organização e não somente o fato de se obter um marco inicial de estar certificado para tal. 


\section{Referências}

[1] Boehm, Barry; IN, Hoh. Identifying Quality-Requirement Conflicts.

1.ed. USA : IEEE Software, 1996, march, p 25-35.

[2] Boehm, Barry. Software Model Conflicts and How to Avoid Them.

SBES`98, XII Simpósio Brasileiro de Engenharia de Software, Maringá, Paraná.

1.ed. Brasil : SBC, Tutorial 1998, outubro, 80 p. (http://www.sunset.usc.edu)

[3] Castro, Jaelson; Alencar, Fernanda; Cysneiros, Gilberto, Mylopoulos, John. From Early Requirements Modeled by the I* Technique to Later Requirements Modeled in Precise UML. WER'00, III Workshop de Engenharia de Requisitos

1ed. Brasil : Rio de Janeiro, Anais WER 2000, julho, vol.1, n.1, p 92-108.

[4] Chung, Lawrence; Nixon, Brian A.; Yu, Eric; Mylopoulos, John. Non-functional Requirements in Software Engineering. 1ed. USA : Kluwer Academic Publishers,2000, 439 p.

[5] CMMI Project. CMM Integrated Systems/ Software Engineering. Carnegie Mellon University. Continuous Representation vol.1

1ed. USA : CMU/SEI, Pensylvania, version 0.2b (Public Release DRAFT) 1999.

[6] Cysneiros, Luiz Márcio; Leite, Júlio C.S.P; Sábat Neto, Jaime de Melo. Non-Functional Requirements for Object-Oriented Modeling. WER'00, III Workshop de Engenharia de Requisitos. 1ed. Brasil : Rio de Janeiro, Anais WER 2000, julho, vol.1, n.1, p 109-125.

[7] Doorn, Jorge; Leite, Júlio C.S.P; Kaplan, Gladys N; Hdad, Graciela D.S. Inspección del Lexico Extendido del Lenguaje. WER'00, III Workshop de Engenharia de Requisitos.

1ed. Brasil : Rio de Janeiro, Anais WER 2000, julho, vol.1, n.1,p 70-91.

[8] FOCALPOINT. Prioritizing Requirements: "What we want always exceeds what we can afford". (http://www.focalpoint.se/Metod/e_index.htm)

[9] ISO/IEC, International Standard Organization. JTC1 - Joint Technical Committee. Information Technology - Software Engineering - Product quality ISO/IEC 9000.

1.ed. Geneve : ISO/IEC, 2000.

[10] ISO/IEC, International Standard Organization. JTC1 - Joint Technical Committee. Information Technology - Software Engineering - Product quality ISO/IEC 9126-x. part1 Quality model; part2 External metrics; part3 Internal metrics; part4 Quality in use.

1.ed. Geneve : ISO/IEC, 1996.

[11] ISO/IEC, International Standard Organization. JTC1 - Joint Technical Committee. Information Technology - Software Engineering - Product quality ISO/IEC 14598 (1-6)

1.ed. Geneve : ISO/IEC, 1998.

[12] ISO/IEC, International Standard Organization. JTC1 - Joint Technical Committee. Information Technology - Software Engineering - Lifecycle Process ISO/IEC 12207.

1.ed. Geneve : ISO/IEC, 1995.

[13] Kilov, Haim. Business Specifications, The Key of Successfull Software Engineering. 1.ed. USA : Prentice Hall PTR, Inc. New Jersey 07458, 1999, 301 p.

[14] Leite, Júlio C.S.P. Viewpoints on Viewpoints. ISAW-2 International Workshop on Multiple Perspectives in Software Development. (San Francisco,CA,USA). 1ed. USA : ACM. Joint Proceedings SIGSOFT'96, 1996, p 285-288.

[15]Pinheiro, Francisco A.C. Formal and Informal Aspects of Requirements Tracing. WER'00, III Workshop de Engenharia de Requisitos. 1ed. Brasil : Rio de Janeiro, Anais WER 2000, julho, vol.1, n.1, p 38-53. 
[16] PMBOK, A Guide to the Project Management Body of Knowledge. 1ed. USA : PMI. Project Management Institute. Four Campus Boulevard, Newton Sq, Pennsylvania USA, 2000, 216p.

[17] Ryan, Kevin. Requirements Engineering - getting value for money. SBES'98, XII Simpósio Brasileiro de Engenharia de Software, Maringá, Paraná. 1.ed. Brasil : SBC Sociedade Brasileira de Computação, Tutorial, 1998,outubro,55 p.

[18] Sommerville, Ian; Sawyer, Pete. Requirements Engineering, A good practice guide. 1ed. UK : Chichester, Baffins Lane, John Wiley \& Sons Ltd. 1997, 391p.

[19] Zanlorenci, Edna P; Burnett, Robert C. Modelo para qualificação da fonte de informação cliente e de requisito funcional. WER'98 de Engenharia de Requisitos.

SBES'98, 1ed. Brasil : SBC, Anais WER 1998, outubro, vol.1, n.1, p 39-48.

[20] Zanlorenci, Edna P; Burnett, Robert C. Descrição e Qualificação de Requisitos: Um Modelo Aplicável à Análise e Validação da Informação. 1ed. Brasil : Pontifícia Universidade Católica do Paraná - PUCPR. Dissertação de Mestrado. 1999, julho, 229p.

[21] Zanlorenci, Edna P; Burnett, Robert C. Ferramenta de Apoio aos Processos de ER, nas Fases de Projeto. WER'00, III WS de Engenharia de Requisitos.

1ed. Brasil : Rio de Janeiro, Anais WER 2000, julho, vol.1, n.1, p 181-193.

[22] Zanlorenci, Edna P; Burnett, Robert C. O Ciclo de Vida de Requisitos, nas Fases de Projeto. I WS Engenharia de Software. 1ed. Chile : Punta Arenas, Anais JCC 2001, novembro, vol.1, n.1, CD.

[23] Zanlorenci, Edna P; Burnett, Robert C. O Tratamento da Informação (Requisitos no Ciclo de Vida do Produto) Caso Prático: Sistema de Informações de Previdência. WER'01, IV WS de Engenharia de Requisitos.

1ed. Argentina : Buenos Aires, Anais WER 2001, novembro, vol.1, n.1, p 55-73. 